\title{
Linearizing Control of Biogas Flow Rate and Quality
}

\author{
Khadidja Chaib Draa, Holger Voos \\ Interdisciplinary Centre for Security, Reliability \\ and Trust (SnT) - Université du Luxembourg \\ L-1359, Luxembourg-Kirchberg, Luxembourg \\ khadidja.chaibdraa/holger.voos@uni.lu
}

\author{
Marouane Alma, Mohamed Darouach, \\ CRAN-CNRS UMR 7039 \\ Université de Lorraine, IUT de Longwy \\ 54400, Cosnes et Romain, France \\ marouane.alma/mohamed.darouach@univ-lorraine.fr
}

\begin{abstract}
In this paper, we propose to control the quantity and quality of the produced biogas from the anaerobic digestion of organic matter, digested in either a continuous stirred tank reactor or a fixed bed digester. This is motivated by the aim of providing the power grid with a stable amount of energy despite fluctuations in the treated waste concentration and composition. Therefore, we apply the linearizing control principe to a two step (acidogenesis-methanogenesis) mass balance non linear model, all with the introduction of two new control inputs reflecting the addition of stimulating substrates (acetate and alkalinity). To show the performance of the synthesized control laws we simulate the process under an organic shock load feeding.

Index Terms-Anaerobic Digestion; Linearized Control; NonLinear Models.
\end{abstract}

\section{INTRODUCTION}

The Anaerobic Digestion (AD) is a complex biological process involving different interacting microorganisms which degrade anaerobically the organic matter to biogas. The later is a mixture of different gaseous but often assumed to be mainly composed of methane (CH4) and carbon dioxide (CO2).

Usually in Biogas Plants (BPs), the produced biogas is burned and converted by co-generation engines to heat and electricity for local use or for use via energy distribution grids. However, it may also be used as a vehicle fuel when it is rich in methane. Thus, the more produced biogas is rich in methane the more waste valorisation is gainful. Moreover, the engines useful lives depends on the produced biogas quality.

Furthermore, it has been reported in [5] that even if the produced biogas is not converted to an other type of energy and is simply released in the air, the amount of unburned hydrocarbon emissions decreases when the biogas is rich in methane. Therefore, the biogas quality control is important whether for economical or ecological reasons.

However, the biogas production control is a challenging task since the AD process involves different survival microorganisms having different optimal life conditions and their functioning is strongly affected by the feedstock composition and concentration, and the operating conditions [3]. Moreover, modeling the full AD process results in complex and high order models not suitable for classical control, we can cite the ADM1 (for Anaerobic Digestion Model 1) [4] for instance.
Therefore, one crucial step to do before dealing with the control part is to get an adequate model suitable for a realistic control implementation. For this end, we first modify some existing models in the literature [4], [6] and [7] as described in Sec. (II). Then, being motivated by the aim of providing the power grid with a stable amount of energy despite fluctuations in the incoming waste concentration and composition, we propose to apply the linearizing control principe [1] to the proposed model in order to regulate the methane flow rate in Sec. (III). Moreover, due to the biogas quality importance we control it in Sec. (IV) through the control of $\mathrm{CO}_{2}$ percentage in the produced biogas. Finally, before concluding the paper in Sec. (VI) we validate, by simulations in Sec. (V), the proposed control laws despite strict fluctuations in the feedstock characteristics.

\section{MODEL DESCRIPTION}

Due to the complexity of the $\mathrm{AD}$ process and the lake of measurement devices many models have been built in the literature for only specific applications. Moreover, most of the models built for control purpose [1], [2], [4] and [7], allow it only by acting on the waste feeding rate to the digester. However, sometimes the infrastructure of the biogas plant does not allow enough storage, or the plant is constrained to treat a certain amount of waste per day. Therefore, we have slightly modified the models described in [4] and [6] by adding two new control inputs reflecting the addition of stimulating acetate and alkalinity to the digester. The considered two limiting steps in the model are:

1) Acidogenesis with reaction rate $r_{1}=\mu_{1} X_{1}$ :

$$
k_{1} S_{1} \stackrel{r_{1}}{\rightarrow} X_{1}+k_{2} S_{2}+k_{4} C O_{2}
$$

2) Methanogenesis with reaction rate $r_{2}=\mu_{2} X_{2}$ :

$$
k_{3} S_{2} \stackrel{r_{2}}{\rightarrow} \mathrm{X}_{2}+k_{5} \mathrm{CO}_{2}+k_{6} \mathrm{CH}_{4}
$$

Modelled by the following equations:

$$
\begin{aligned}
& \dot{S}_{1}=-k_{1} \mu_{1}\left(S_{1}\right) X_{1}+D_{1} S_{1 i n}-D S_{1} \\
& \dot{X}_{1}=\left(\mu_{1}\left(S_{1}\right)-\alpha D\right) X_{1} \\
& \dot{S}_{2}=k_{2} \mu_{1}\left(S_{1}\right) X_{1}-k_{3} \mu_{2}\left(S_{2}\right) X_{2}+D_{1}\left(S_{2 i n}+S_{0}\right)-D S_{2} \\
& \dot{X}_{2}=\left(\mu_{2}\left(S_{2}\right)-\alpha D\right) X_{2} \\
& \dot{C}=k_{4} \mu_{1}\left(S_{1}\right) X_{1}+k_{5} \mu_{2}\left(S_{2}\right) X_{2}+D_{1} C_{i n}-D C-Q_{c} \\
& \dot{Z}=D_{1} Z_{i n}+D_{2} Z_{0}-D Z
\end{aligned}
$$


where, $S_{1}$ is the concentration of the organic matter to be digested. $X_{1}$ is the concentration of the acidogenic bacteria responsible for the organic matter degradation. $S_{2}$ is the VFA concentration which is supposed to behave like pure acetate and $X_{2}$ is the methanogenic bacteria concentration. Then, $Z$ represents the alkalinity which is the sum of acetate and bicarbonate $(\mathrm{Bic})$ concentrations:

$$
Z=B i c+S_{2}
$$

The inorganic carbon concentration in the digester $C$ is assumed to be the sum of the dissolved carbon dioxide $\left(\mathrm{CO}_{2}\right)$ and bicarbonate:

$$
\left\{\begin{array}{l}
C=C O_{2}+B i c \\
k_{b}=\frac{\left[H^{+}\right] B i c}{C O_{2}} \\
p H=-\log _{10}\left(K_{b} \frac{C O_{2}}{B i c}\right)
\end{array}\right.
$$

where $k_{b}$ is the acidity constant of bicarbonate. In Eqs. (3), $Q_{c}$ is the $\mathrm{CO}_{2}$ gas flow rate:

$$
Q_{c}=\frac{R T \gamma C O_{2}}{P_{T}+R T \gamma\left(K_{H} P_{T}-C O_{2}\right)} Q_{M}
$$

where $\gamma$ is a dimensionless parameter introduced by Hess [4] in order to reduce the expression complexity between the dissolved and the gaseous carbon dioxide. While $Q_{M}$ is the methane flow rate which is supposed to be proportional to the reaction rate of the mathanogenesis due to its low solubility:

$$
Q_{M}=k_{6} \mu_{2}\left(S_{2}\right) X_{2}
$$

Moreover, the growth rates of acidogenic bacteria $\mu_{1}\left(S_{1}\right)$ and methanogenic bacteria $\mu_{2}\left(S_{2}\right)$ are supposed to be of type Monod and Haldane, respectivaly:

$$
\begin{gathered}
\mu_{1}\left(S_{1}\right)=\mu_{1 \max } \frac{S_{1}}{S_{1}+k_{s 1}} \\
\mu_{2}\left(S_{2}\right)=\mu_{2 \max } \frac{S_{2}}{S_{2}+k_{s 2}+\frac{S_{2}^{2}}{k_{I 2}}}
\end{gathered}
$$

In all previous described dynamics, we referred to the respective incoming concentrations by $X_{i n}$. Moreover, $k_{i}$ are the yield coefficients defined in Table (I) with the other parameters. The dilution rate $D_{1}$ is calculated by dividing the flow rate of the incoming waste and the added stimulating acetate with concentration $S_{0}$ by the digester volume. Whereas, $D_{2}$ is calculated by dividing the flow rate of the added stimulating alkalinity with concentration $Z_{0}$ by the digester volume. Morover, for the mass balance we take $D=D_{1}+D_{2}$. Finally, the parameter $\alpha$ reflects the proportion of the dilution rate for bacteria ( $\alpha=0$ corresponds to an ideal fixed bed reactor and $\alpha=1$ corresponds to a ideal Continuous Stirred Tank Reactor (CSTR) [7]).

\section{Methane Flow Rate Control}

The produced energy from biogas is proportional to the burned quantity of methane [3]. Thus, we apply the linearizing control principe to the model Eqs. (3) in order to control the
$\mathrm{CH}_{4}$ flow rate. Therefore, we first select a first order reference model for the regulation error:

$$
\frac{d\left(Q_{M}^{*}-Q_{M}\right)}{d t}+\lambda\left(Q_{M}^{*}-Q_{M}\right)=0
$$

where $Q_{M}^{*}$ is the reference value of the $\mathrm{CH}_{4}$ flow rate which allows the biogas plant to satisfy the requested energy from the power grid and $\lambda$ is a design parameter which depends on the desired close loop performance. Now, lest's assume that the biogas plant should provide the power grid a constant amount of electricity despite the inlet concentration fluctuations and thus $Q_{M}^{*}$ is constant which implies that:

$$
\frac{d Q_{M}}{d t}=\lambda\left(Q_{M}^{*}-Q_{M}\right)
$$

Moreover, using Eq. (7) the time derivative of $Q_{M}$ is given by:

$$
\frac{d Q_{M}}{d t}=k_{6} \frac{d \mu_{2}\left(S_{2}\right)}{d t} X_{2}+k_{6} \mu_{2}\left(S_{2}\right) \frac{d X_{2}}{d t}
$$

Furthermore, the dynamic of $\mu_{2}\left(S_{2}\right)$ is given by:

$$
\frac{d \mu_{2}\left(S_{2}\right)}{d t}=\frac{d \mu_{2}\left(S_{2}\right)}{d S_{2}} \frac{d S_{2}}{d t}
$$

After some small mathematical manipulations we get:

$$
\begin{aligned}
\frac{d \mu_{2}\left(S_{2}\right)}{d t}= & \frac{k_{s 2} k_{I 2}-S_{2}^{2}}{k_{I 2} \mu_{2 \max }} \frac{\mu_{2}^{2}\left(S_{2}\right)}{S_{2}^{2}} \\
& \left(D_{1}\left(S_{2 i n}+S_{0}\right)-D S_{2}+k_{2} \mu_{1}\left(S_{1}\right) X_{1}-\frac{k_{3}}{k_{6}} Q_{M}\right)
\end{aligned}
$$

Now, lest's the variable $\theta$ be:

$$
\theta=\frac{k_{s 2} k_{I 2}-S_{2}^{2}}{k_{I 2} \mu_{2 \max }} \frac{\mu_{2}\left(S_{2}\right)}{S_{2}^{2}}
$$

Hence, substituting Eq. (15) in Eq. (14) and using Eqs. (7), (11) and Eq. (12) we get:

$$
\begin{aligned}
& \frac{\lambda\left(Q_{M}^{*}-Q_{M}\right)}{\theta Q_{M}}=D_{1}(\left.S_{2 i n}+S_{0}\right)-D S_{2}+k_{2} \mu_{1}\left(S_{1}\right) X_{1} \\
&-\frac{k_{3}}{k_{6}} Q_{M}+\frac{1}{\theta}\left(\mu_{2}\left(S_{2}\right)-\alpha D\right)
\end{aligned}
$$

Finally, from Eq. (16) many control laws can be synthesized. But since the plant is constrained to treat a fixed amount of waste per day the dilution rate $D_{1}$ is kept constant. Moreover, the operator has no control in the incoming waste concentrations, so we act on the added stimulating acetate concentration $S_{0}$ :

$$
\begin{aligned}
S_{0}= & \frac{1}{D_{1}} \times \\
& \left(\begin{array}{rl}
\frac{\lambda\left(Q_{M}^{*}-Q_{M}\right)}{\theta Q_{M}}+D S_{2}-k_{2} \mu_{1}\left(S_{1}\right) X_{1}+ \\
\left.\frac{k_{3}}{k_{6}} Q_{M}-\frac{1}{\theta}\left(\mu_{2}\left(S_{2}\right)-\alpha D\right)\right)-S_{2 i n}
\end{array}\right.
\end{aligned}
$$

We notice that the control Eq. (17) does not require measurement of $S_{1 i n}$ and all its variables are even known or commonly measured in a real life operating plant except the acidogenesis bacteria concentration which is costly and complex to measure. 


\section{Carbon Dioxide Percentage Control}

In the previous section we proposed to control the flow rate of methane by adding a stimulating acetate. However, acids accumulation causes a $p H$ breakdown when the buffering capacity of the reactor if low. Therefore, if no countermeasure is taken the methanogenisis is inhibited and the biogas production will decrease till the point where it is zero. Moreover, it has been reported in [4] that the dissolved and gaseous $\mathrm{CO}_{2}$ are strongly related by the $p H$ and an increase of the reactor alkalinity (buffering capacity) enhances the biogas quality. Therefore, we propose to control the biogas quality by acting on the dilution rate $D_{2}$ of the added stimulating alkalinity with concentration $Z_{0}$. Thus, using the linear relation between the quality of biogas $\left(\% \mathrm{CO}_{2}\right)$ and the dissolved $\mathrm{CO}_{2}$ given by Hess et all [4]:

$$
\% \mathrm{CO}_{2}=\frac{R T \gamma}{P_{T}+R T \gamma K_{H} P_{T}} C O_{2}
$$

we obtain the reference value of the dissolved carbon dioxide $\left(\mathrm{CO}_{2}^{*}\right)$ which corresponds to the desired biogas quality $\% \mathrm{CO}_{2}^{*}$ ) and consequently using Eq. (4) and Eqs. (5) we deduce the set point of the alkalinity in the reactor $Z^{*}$ :

$$
Z^{*}=\left(\frac{K_{b}}{10^{-p H}}\right) C O_{2}^{*}+S_{2}
$$

We highlight an important feature, the alkalinity reference value is updated in Eq. (19) with respect to the real state of the reactor $\left(S_{2}\right)$.

Now, once again we apply the principe of linearizing control to control the biogas quality. Therefore, we select a first order reference model for the regulation error:

$$
\frac{d\left(Z^{*}-Z\right)}{d t}+\beta\left(Z^{*}-Z\right)=0
$$

which also implies that (since $\frac{d Z^{*}}{d t}=0$ because $Z^{*}$ is constant):

$$
\frac{d Z}{d t}=\beta\left(Z^{*}-Z\right)
$$

where $\beta$ is a design parameter which depends on the desired close loop performance. Thus, using the dynamic equation of $Z$ described in Eqs. (3) we get:

$$
\beta\left(Z^{*}-Z\right)=D_{1} Z_{\text {in }}+D_{2} Z_{0}-D Z
$$

Finally, the same remarque as previous (for Eq. (16)) many control laws can be synthesized. However, for an easy implementation of the control low in a real life operating plant we suppose that the added stimulating alkalinity concentration is constant. Thus, the control is designed for the dilution rate of the added stimulating alkalinity:

$$
D_{2}=\frac{\beta\left(Z^{*}-Z\right)-D_{1}\left(Z_{i n}-Z\right)}{Z_{0}-Z}
$$

However, in order to avoid the washout of bacteria $D_{2}$ can range only in an admissible interval such that $0 \leq D_{1}+D_{2}<$ $D_{\max }$.

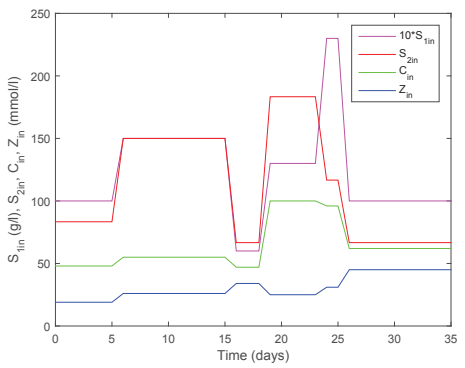

Fig. 1: Incoming concentrations.

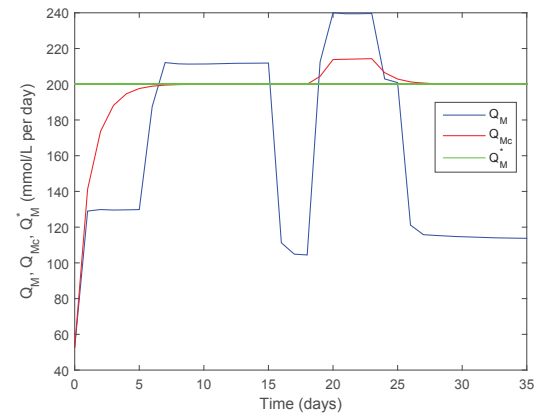

Fig. 2: Controlled and uncontrolled methane flow rate.

\section{Simulation Results}

In order to estimate the performance of the proposed control laws for regulating the $\mathrm{CH}_{4}$ flow rate and the $\mathrm{CO}_{2}$ percentage in the produced biogas despite fluctuations in the incoming waste concentrations, we have chosen to vary the later in a wide range of realistic expected values including the shock load case Fig. (1). Moreover, in what concerns the type of the digester it is considered to be neither a perfect CSTR nor a perfect fixed bed reactor so $\alpha=0.5$. As well, we have fixed the dilution rate $D_{1}=0.6\left(d a y^{-1}\right)$, the maximum concentration of the added stimulating acetate $S_{0 \max }=150$ $(\mathrm{mmol} / \mathrm{l})$ and the concentration of the added stimulating alkalinity and $Z_{0}=180(\mathrm{mmol} / \mathrm{L})$. Moreover, to avoid the bacteria washout the overall dilution rate $D$ is limited by $D_{\max }=\frac{\mu_{2 \max }}{\alpha}$. Besides, for the desired closed loop performance; $\lambda=0.8$ and $\beta=0.05$.

The simulation results are presented in Figs. (2), (3), (4) and Fig. (5), we notice that for simulations the parameter values given in Table. (I) are approximations taken from the literature.

Indeed, Fig. (2) shows that the system tracks very well the reference $Q_{M}^{*}$ although the severe fluctuations of the inlet concentrations. As well, we see from Fig. (3) that there is no accumulation of acids and no inhibition of the methanogenic bacteria, on the contrary the later concentration behaves very well where it keeps increasing despite the shock loading applied to the system. On the other hand, the biogas quality control is not satisfactory as it can be seen from Fig. (4) which may be due to the constraint posed on $D$ during the simulations (since it intervenes in all the system dynamics) and the a priori fixed value $Z_{O}$. However, the controlled biogas 


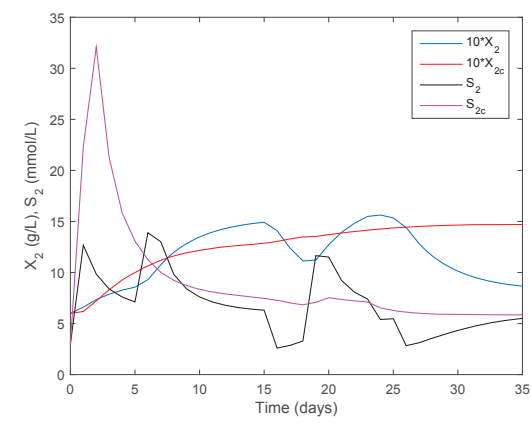

Fig. 3: Controlled and uncontrolled concentrations.

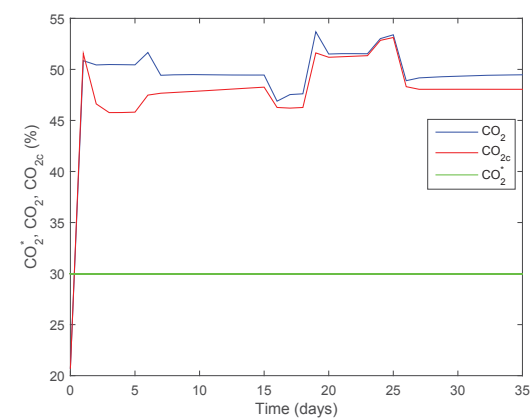

Fig. 4: Controlled and uncontrolled biogas quality.

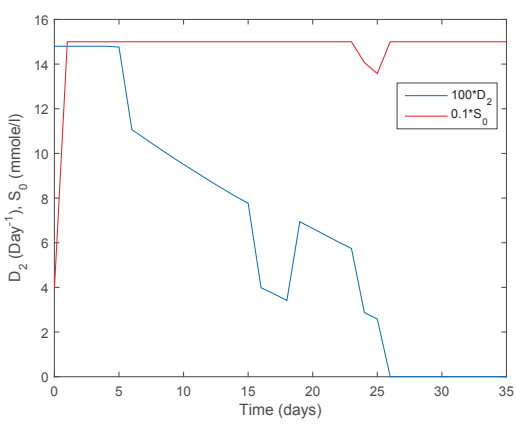

Fig. 5: Control inputs.

quality is better than the uncontrolled one. Finally, from Fig. (5) we see that the inputs $D_{2}$ and $S_{0}$ do not vary abruptly which make there implementation in a real life operating plant feasible.

\section{CONCLUSION}

In this paper, we proposed to control the quantity and quality of the produced biogas from the anaerobic digestion of organic matter. Therefore, first we have slightly modified some existing models in the literature by including the addition of stimulating acetate and alkalinity. Then, we have designed two linearizing control laws for both the $\mathrm{CH}_{4}$ flow rate and the $\mathrm{CO}_{2}$ percentage in the produced biogas control. This all was motivated by the aim of introducing the biogas plants in a virtual power plant and provide the power grid with a stable amount of energy despite fluctuations in the treated waste characteristics.

The simulation results for the $\mathrm{CH}_{4}$ flow rate control have shown suitable performances although the sever variations of the incoming waste concentration and composition. However, the quality of the produced biogas could not reach the exact reference value due to the saturation made on the dilution rate to avoid the bacteria washout, and the a priori fixed values of the added stimulating substrates.

In the next future, we target to enhance the biogas quality control and to use only commonly done measurements in a real life operating plant while controlling the biogas quantity and quality.

TABLE I: Nomenclature

\begin{tabular}{|c|c|c|c|}
\hline Acronyms & Definition & 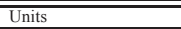 & Values \\
\hline$\alpha$ & Proportion of dilution rate for bacteria & $m m o l / l$ & 0.5 \\
\hline$k_{1}$ & Yield for substrate degradation & $g /\left(g\right.$ of $\left.X_{1}\right)$ & 42.1 \\
\hline$k_{2}$ & Yield for VFA production & $\mathrm{mmol} /\left(\mathrm{g}\right.$ of $\left.X_{1}\right)$ & 116.5 \\
\hline$k_{3}$ & Yield for VFA consumption & $\mathrm{mmol} /\left(g\right.$ of $\left.X_{2}\right)$ & 268 \\
\hline$k_{4}$ & Yield for $\mathrm{CO}_{2}$ production & $\mathrm{mmol} / \mathrm{g}$ & 100 \\
\hline$k_{5}$ & Yield for $\mathrm{CO}_{2}$ production & $\mathrm{mmol} / \mathrm{g}$ & 375 \\
\hline$k_{6}$ & Yield for $C h_{4}$ production & $\mathrm{mmol} / \mathrm{g}$ & 453 \\
\hline$\mu_{1 \max }$ & Maximum acidogenic bacteria growth rate & $1 / d a y$ & 1.25 \\
\hline$\mu_{2 \max }$ & Maximum methanogenic bacteria growth rate & $1 / d a y$ & 0.74 \\
\hline$k_{s 1}$ & Half saturation constant associated with $S_{1}$ & $g / l$ & 0.41 \\
\hline$k_{s 2}$ & Half saturation constant associated with $S_{2}$ & $\mathrm{mmol} / \mathrm{l}$ & 8.42 \\
\hline$K_{I 2}$ & Inhibition constant associated with $S_{2}$ & $\mathrm{mmol} / \mathrm{l}$ & \\
\hline$K_{b}$ & Acidity constant of bicarbonate & $\mathrm{mol} / \mathrm{l}$ & $6.5 \times 10^{-7}$ \\
\hline$K_{H}^{0}$ & Henry s constant & mmole/(l.atm) & \\
\hline$R^{M}$ & Gas constant & L.atm $/($ K.mol $)$ & 0.0821 \\
\hline$P_{T}$ & Total preasure & atm & 1.013 \\
\hline $\mathrm{T}$ & Temperature & Kelvin & 308 \\
\hline$\gamma$ & dimonsionless parameter introduced by Hess [4] & - & 0.025 \\
\hline
\end{tabular}

\section{REFERENCES}

[1] G. Bastin and D. Dochain. On-line Estimation and Adaptive Control of Bioreactors. Process measurement and control. Elsevier, 1990.

[2] B. Benyahia. Modélisation et Observation des Bioprocédés à Membranes: Application à la Digestion Anaérobie. $\mathrm{PhD}$ thesis, Université de Tlemcen, Algérie et Univesité de Montpelier 2, France, 2012.

[3] D. Bernhard. Process Monitoring in Biogas Plants. IEA Bioenergy, 2013.

[4] J. Hess. Modélisation de la Qualité du Biogas Produit par un Fermenteur Méthanogène et sa Stratégie de Régulation en Vue de sa Valorisation. $\mathrm{PhD}$ thesis, Universit de Nice - Sophia Antipolis, 2008.

[5] J. Huang and R.J. Crookes. Assessment of Simulated Biogas as a Fuel for the Spark Ignition Engine. Fuel, 77(15):1793 - 1801, 1998.

[6] M. Darouach K. Chaib Draa, H. Voos and M.Alma. A Formal Modeling Framework for Anaerobic Digestion Systems. 2015. Uksim-Amss, Cambridge.

[7] D. Dochain A. Genovesi O. Bernard, Z. Hadj-Sadok and J.P. Steyer. Dynamical Model Development and Parameter Identification for an Anaerobic Wastewater Treatment Process. Biotechnology and Bioengineering, 75(4):424 - 438, 2001. 